\title{
Due tecnicismi artistico-architettonici di origine francese: orifiamma e vaso orifiamma
}

Matteo Mazzone

PUBBLICATO: 18 DECEMBER 2020

\section{Quesito:}

Alcuni dei nostri lettori ci chiedono informazioni riguardo alla corretta forma del plurale e all'etimologia di orifiamma, nonché al significato che la parola assume in ambito artistico e architettonico.

\section{Due tecnicismi artistico-architettonici di origine francese: orifiamma e vaso orifiamma} racciare e ripercorrere il cammino evolutivo del termine orifiamma permette di comprendere a pieno quei meccanismi formali e semantici, spesso oscurati dal passare del tempo, che coinvolgono molte delle parole tecnico-scientifiche dellitaliano.

Riprendendo alcune considerazioni già suggerite dai lettori, confermiamo che del significato artistico e architettonico di orifiamma non si riscontrano notizie né nei repertori tecnico-specialistici, né nei dizionari storici dell'italiano, né in quelli dell'uso. Possiamo supporre che la specializzazione terminologica della voce orifiamma, come vedremo meglio in seguito, sia il risultato di un processo di risemantizzazione del senso originale della parola, il cui utilizzo sia rimasto e rimanga ancora oggi confinato alla terminologia settoriale e specialistica di àmbito artistico e architettonico. Ma cominciamo dal principio.

Se ricerchiamo e analizziamo l'etimologia della voce orifiamma, troviamo negli strumenti lessicografici soluzioni almeno in parte discordanti. Che si tratti di un francesismo pare piuttosto sicuro: più controverse, invece, risultano le posizioni degli studiosi circa il significato originario dell'etimo francese. Secondo il DEI la forma è da considerarsi un prestito romanzo dal francese oriflame, derivato a sua volta dall'antico francese oriflambe, traduzione del latino aureaflamma. Una posizione differente si riscontra nel DELI, che data il prestito dall'antico francese orieflambe al Io8o, analizzandolo come un composto lessicale di orie 'aurea' e flambe 'framma, o giglio', derivato dal diminutivo latino flammula. Il DELI, però, segnala che l'accezione di oriflambe/orieflambe 'framma, giglio dorato' "è una successiva interpretazione (non era un vessillo dorato, bensi rosso)". Si intravede cosi una nuova ipotesi etimologica, che prevede di considerare l'antico francese orieflambe come traduzione dell'espressione latina aurita flammula, cioè "piccola fiamma = bandiera dentellata = a orecchie". Nell'Etimologico di Alberto Nocentini orifiamma è ricondotto al francese oriflamme, composto di flamme, dal latino medievale flamma 'bandiera', e di orie, continuazione del latino aurěus 'd'oro'. Di parere ancora differente era stato Bruno Migliorini, che nel suo articolo A proposito di oriflamme e orifiamma (1975) sostiene la derivazione dal latino labari flamma o laurea flammula, espressioni che assumerebbero il significato di 'vittoria, bandiera vittoriosa'.

Tra i vari significati assunti, sia i vocabolari storici dell'italiano sia quelli della lingua contemporanea sono concordi nel definire il sostantivo orifiamma come 'il vessillo militare di colore rosso cosparso di stelle o fiamme dorate': il GDLI mette a lemma la voce, marcandola come antica e letteraria, con il significato di 'bandiera di seta rossa con stelle ricamate e framme d'oro dipinte, che era posta 
nell'abbazia di Saint-Denis e usata come insegna militare dai re di Francia dal sec. XII al sec. XIV'. Anche il più ricco dizionario dell'italiano contemporaneo, il GRADIT di Tullio De Mauro, registra la voce inserendo una definizione che, seppur con qualche piccolissima modifica nella sua struttura, appare simile a quella del GDLI appena ricordata. Inoltre, la definizione è preceduta dalla sigla TS, che specifica la marca d'uso della parola, precisando a quale strato del lessico italiano appartiene orifiamma con il senso di bandiera militare: quello tecnico-specialistico di àmbito storico. Stando, inoltre, agli esempi che si rintracciano nel GDLI, orifiamma col tale significato è attestato dalle origini della nostra letteratura sino al Novecento (l'ultimo esempio citato è della scrittrice d'origine toscana Jolena Baldini). A voler esser più precisi, la prima testimonianza della voce sino a oggi conosciuta, come si riscontra nel repertorio dei testi antichi TLIO, risale al Paradiso di Dante del I32r: il poeta usa orifiamma con una valenza che ancora oggi suscita perplessità e incertezza: lo stesso TLIO ne suppone il senso di 'fiamma d'oro', ma aggiunge un punto interrogativo, mantenendo un margine di dubbio nella sua definizione, mentre sia il GDLI sia il GRADIT la definiscono come parte dell'ordinamento del Paradiso dantesco, in particolare 'il gradino più alto e luminoso dell'Empireo al centro del quale è situata Maria'. Infatti, il significato di orifiamma come 'vessillo militare', solo apparentemente legato a un contesto profano come quello militare, si applica anche a quello sacro: da sempre, nella simbologia religiosa e cristiana, la frammella d'oro è emanazione della luce divina, redentrice e purificatrice contro le tenebre oscure del male.

Cosi, sacro e profano si uniscono nella rappresentazione dell'Apparizione della Croce a Costantino del I606 dipinta dal pittore Grazio Cossali e collocata nella cappella delle Sante Croci del duomo vecchio di Brescia (di cui qui sotto, alla figura I, si ripropone il particolare della Croce del Campo o Croce dell'Orifiamma): sotto la croce si trova il labaro rosso purpureo, ossia lo stendardo dell'imperatore Costantino, al centro del quale si intravede chiaramente la sigla monogrammatica di Cristo (chrismon: 首), circondata da una corona di foglie d'alloro dorate; sullo sfondo, gruppi di frammelle tremolanti. Rimanendo in àmbito religioso, nella figura 2 è riprodotto l'affresco dell'orifiamma di San Bernardino da Siena, conservato nella chiesa di Santa Maria degli Angioli di Lugano: dal bordo esterno della circonferenza, entro cui è inscritto il trigramma di Cristo (YHS), si diramano dodici framme rossodorate, che riappaiono nitidamente in due triadi poste nel lato destro della stessa immagine, su entrambi i margini.

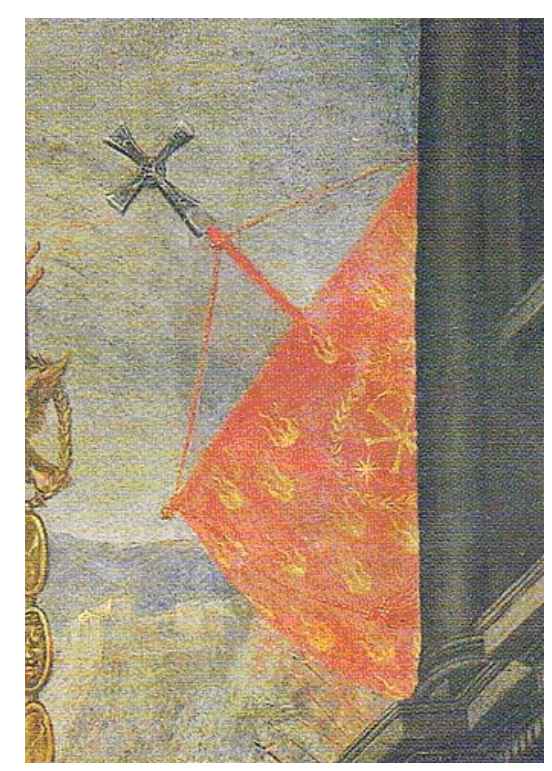

Fig. I: dettaglio dell'Apparizione della Croce a Costantino (Grazio Cossali, Duomo vecchio, Brescia), it.wikipedia.org 


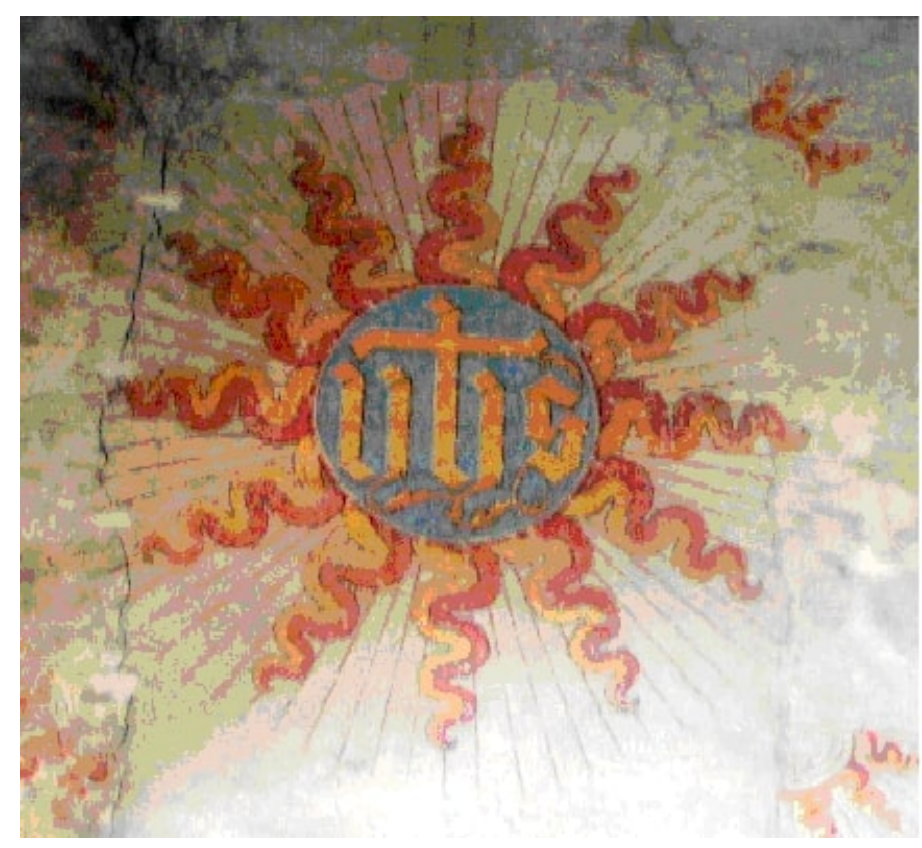

Fig. 2: Orifiamma di san Bernardino dell'affresco nella chiesa di Santa Maria degli Angeli (Lugano), it.wikipedia.org

Nella figura I, orifiamma designa sicuramente il labaro rosso ornato con piccole fiamme (come quelle che circondano la corona dorata d'alloro entro cui è inscritta la sigla di Cristo). Nella figura 2, invece, è preponderante il valore di orifiamma come motivo decorativo: la transizione dal senso principale di 'vessillo, stendardo, labaro purpureo cosparso di framme dorate' a quello di 'tipo di abbellimento, ornamento pittorico' sembra essere avvenuta. Vediamo, adesso, di spiegare come sia nata questa nuova accezione della voce.

Pare ovvio che dal passaggio semantico dalla prima alla seconda accezione sia intervenuto, oltre a una restrizione per sineddoche del valore semantico più ampio di orifiamma, anche un processo di risemantizzazione: in breve, questo processo - che è molto frequente nelle lingue specialistiche e scientifiche - consiste nel rimpiego di parole, d'uso comune o appartenenti a un determinato lessico specialistico, alle quali è fatto corrispondere un nuovo significato tecnico. Così orifiamma, da termine d'àmbito storico con significato di bandiera rossa tempestata di frammelle d'oro, si trasforma in tecnicismo del lessico artistico, designando una tipologia di decorazione che ha per oggetto le sole piccole fiamme dorate cosparse su tutta la superficie pittorica. Si comprenderà, dunque, che il secondo significato è, al tempo stesso, legato imprescindibilmente al primo, ma ne diventa indipendente.

Come già ricordato, il senso di orifiamma quale 'tipologia di decorazione pittorica' non è attestato dai dizionari tecnici né, più in generale, dai repertori lessicografici dellitaliano: fa eccezione il solo GDLI, che registra anche questa sfumatura semantica, citando un unico esempio, novecentesco, tratto dalla lirica di piena atmosfera decadentista Suprema quies, datata al I9o3, del poeta Guido Gozzano, e di cui qui riportiamo i versi che più ci interessano:

Quei lumi/ rischiarano il silenzio sepolcrale: / allineati stan nello scaffale / mille volumi / che alluminava un mastro fiorentino / d'orifiamme e d'armille in cento nodi.

Convincente risulta l'interpretazione del sostantivo orifiamme come 'tipologia di decorazione': Gozzano fa riferimento ai "mille volumi" riccamente decorati e miniati per mano di un "mastro 
fiorentino" - come suggerisce proprio l'azione espressa dal verbo "alluminava" per "miniava" - con "armille", ossia braccialetti o coroncine, e piccole fiammelle.

La storia della parola orifiamma rivela un ulteriore sviluppo semantico, ora in àmbito architettonico. Se osserviamo il particolare, tratto dalla stampa settecentesca dell'architetto e vedutista Giuseppe Vasi, della chiesa di San Giacomo (figura 3), un tempo ubicata nella Piazza Scossacavalli (demolita in epoca fascista per la costruzione dell'attuale via della Conciliazione), noteremo, sia a destra che a sinistra del timpano, un vaso che poggia all'estremità del tetto, quasi in corrispondenza dei due pilastri angolari della struttura architettonica.

Si tratta di un vaso orifiamma o più semplicemente di un'orifiamma lapidea o di ceramica, una specie di orcio, di urna o di grosso vaso festonato e sormontato da una fiamma e utilizzato come ornamento architettonico che corona il vertice e gli angoli di timpani, frontoni o fastigi (come cupole, guglie, lanterne, tetti, ecc.) di edifici sacri o profani. Per una riproduzione più efficace si veda la figura 4 , che rappresenta un vaso acroteriale con orifiamma, conservato presso la Villa del Padergnone a Zanica, in provincia di Bergamo (ringrazio per l'immagine concessa l'amico e collega Lorenzo Mascheretti) e databile al XVIII-XIX secolo.

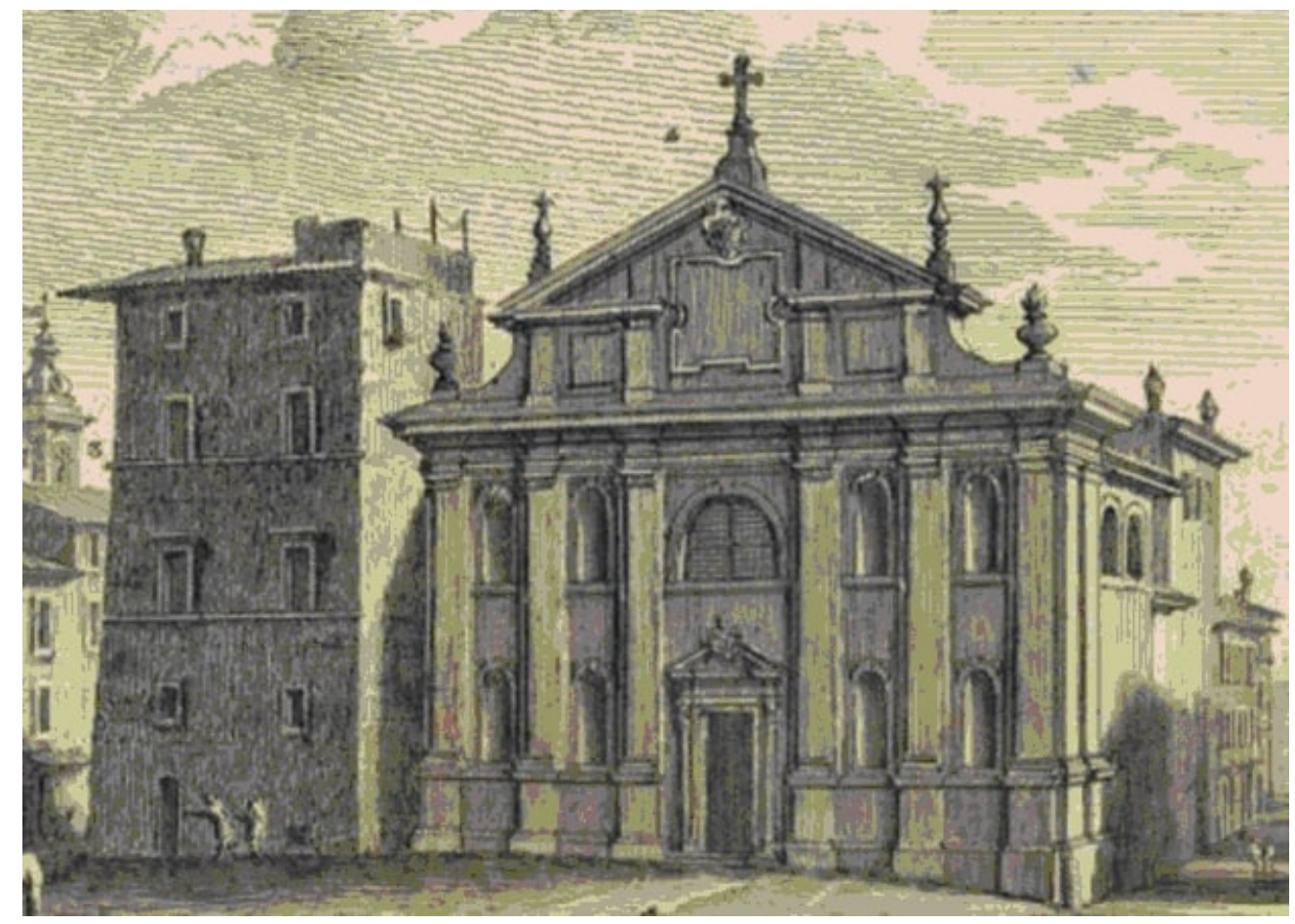

Fig. 3: Giuseppe Vasi, veduta della chiesa di San Giacomo, it.wikipedia.org 


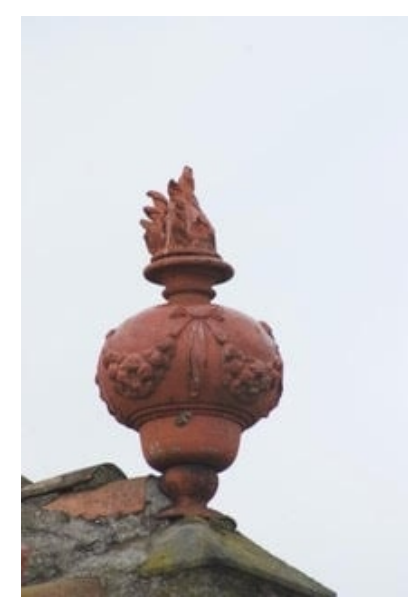

Fig. 4

Ecco, dunque, che sia il composto vaso orifiamma sia orifiamma si caricano di un'ulteriore valenza semantica e acquistano un nuovo significato rispetto a quello originario di orifiamma quale 'vessillo, bandiera purpurea ornata di fiammelle dorate', oppure 'motivo decorativo e pittorico'.

Occorre ancora una breve precisazione grammaticale: l'espressione vaso orifiamma è una composizione lessicale, come angolo cottura, mobile bar, nave traghetto. Si tratta, infatti, della giustapposizione di due sostantivi in cui il secondo elemento (orifiamma) determina, integra e precisa parte del significato del primo, detto testa (vaso): in tal caso, il plurale del secondo nome resta invariato. Dunque, al maschile singolare si utilizzerà vaso orifiamma, mentre al plurale vasi orifiamma (come angoli cottura, mobili bar, navi traghetto), come testimoniano anche i tre esempi che seguono:

Questa poi è sostenuta da due speroni di taglio netto, dal profilo elegantissimo, su cui sorgono in basso due stelle. Il fastigio è costituito da due vasi orifiamma e dallo stemma degli Aldobrandini eretto su un piedistalluccio stretto da due volute. (Paolo Portoghesi, Borromini nella cultura europea, Roma, Officina, 1964, p. 306)

Il terzo livello sorge su un basamento liscio ed è limitato a tre campate concluse da timpano triangolare. Sui tratti residui appaiono edicole tempestate da vasi orifiamma. (Manfredo Tafuri, Ricerca del Rinascimento: principi, città, architetti, Torino, Einaudi, 1992, p. 151)

Sopra i timpani curvi delle porte laterali si stratificano piani che sospingono i cartocci verso l'osservatore. Le porte laterali sono inserite in edicole a timpani triangolari, con vasi orifiamma corrispondenti alle colonne sottostanti; e la porta centrale, a timpano triangolare, è inserita in una edicola a timpano semicircolare. (Amelio Fara, Bernardo Buontalenti, Milano, Electa, 1995, p. 155)

Quanto al genere del nome, i dizionari etimologici, storici e dell'uso registrano orifiamma come sostantivo femminile, prediligendo per il plurale la forma orifiamme: fa eccezione il GDLI, che annota anche il plurale maschile orifiammi. Come prova dello scarso utilizzo del maschile plurale, vale la pena eseguire una piccola ricerca nel web: se digitiamo la stringa "gli orifiammi" su Google, per le pagine in italiano otteniamo solamente 173 risultati, mentre il numero triplica per la stringa "le orifiamme", salendo a 502 (ricerche effettuate in data 5/5/2020). Dunque, alla luce della norma grammaticale riscontrata negli strumenti lessicografici - confermata anche dalla piccola indagine effettuata nel web -, essendo orifiamma un sostantivo femminile, resta preferibile e consigliabile l'utilizzo della forma plurale le orifiamme, che abbiamo visto usata anche da Gozzano.

Al contrario, orifiamma diventa di genere maschile se riferito a un tipo di pesce; più precisamente, 
secondo la definizione tecnico-specialistica registrata dal GRADIT, il nome può indicare anche la 'denominazione di alcune varietà di pesci del genere Carassio, cosi chiamati per la pinna caudale molto lunga e bipartita'. Conducendo una rapida ricerca sul web, sempre per le pagine in italiano, basterà notare come i contesti in cui si ottengono i 1320 risultati della stringa "un orifiamma" e i Iozo del plurale maschile invariato "gli orifiamma" si riferiscono anche (e soprattutto) a una specie di pesce rosso. Rimane comunque incerto capire se lo sviluppo semantico di orifiamma come 'specie di pesce rosso' sia un caso di polisemia in rapporto al significato primario della voce, ossia quello di 'bandiera rossa, vessillo militare'; ma ancora, si potrebbe pensare a un caso di omonimia, ovvero orifiamma 'specie di pesce rosso' e orifiamma 'bandiera rossa, vessillo militare' sarebbero del tutto indipendenti sul piano del significato, ma coinciderebbero solo per la grafia e per la pronuncia.

Concludendo, la voce orifiamma, dal senso originale e tutt'oggi attestato di 'vessillo militare di colore rosso dei re di Francia cosparso di stelle o framme dorate', si è andata specializzando in àmbito sia artistico sia architettonico, divenendo a tutti gli effetti voce tecnica adoperata a seconda del suo preciso campo di applicazione. Nel primo caso, il termine orifiamma descrive e indica un particolare ornamento pittorico e decorativo a piccole fiammelle dorate, applicato su superfici di varia natura e di diverse dimensioni, come possono essere soffitti, volte, pareti, cornici. Nel secondo caso, invece, il composto vaso orifiamma - talvolta attestato semplicemente come orifiamma - indica un abbellimento architettonico che consiste in un orcio o in un vaso lapideo o ceramico, dal cui coperchio fuoriesce una fiamma; di solito questo oggetto, con funzione di acroterio, è posto come coronamento sulle estremità di fastigi, tetti, cupole, guglie, edicole, timpani, frontoni, pilastri, ecc. Certamente, solo il contesto in cui il termine è inserito può aiutare a disambiguare la sua corretta accezione.

Nota Bibliografica:

- Amelio Fara, Bernardo Buontalenti, Milano, Electa, 1995.

- Bruno Migliorini, A proposito di orifiamme e orifiamma, in "Revue roumaine de linguistique", XX, I975, pp. 543-545.

- Paolo Portoghesi, Borromini nella cultura europea, Roma, Officina, 1964.

- Manfredo Tafuri, Ricerca del Rinascimento: principi, cittá, architetti, Torino, Einaudi, 1992.

\section{Cita come:}

Matteo Mazzone, Due tecnicismi artistico-architettonici di origine francese: orifiamma evaso orifiamma , "Italiano digitale", 2020, XV, 2020/4 (ottobre-dicembre)

DOI: $10.35948 / 2532-9006 / 2020.5438$

Copyright 2020 Accademia della Crusca

Pubblicato con licenza creative commons CC BY-NC-ND 